gr-qc/9310003 Alberta-Thy-47-93

\title{
Self-dual gravity as a two dimensional theory and conservation laws
}

\author{
Viqar Husain \\ Theoretical Physics Institute, University of Alberta \\ Edmonton, Alberta T6G 2J1, Canada.
}

\begin{abstract}
Starting from the Ashtekar Hamiltonian variables for general relativity, the self-dual Einstein equations (SDE) may be rewritten as evolution equations for three divergence free vector fields given on a three dimensional surface with a fixed volume element.

From this general form of the SDE, it is shown how they may be interpreted as the field equations for a two dimensional field theory. It is further shown that these equations imply an infinite number of non-local conserved currents.

A specific way of writing the vector fields allows an identification of the full SDE with those of the two dimensional chiral model, with the gauge group being the group of area preserving diffeomorphisms of a two dimensional surface. This gives a natural Hamiltonian formulation of the SDE in terms of that of the chiral model. The conservation laws using the explicit chiral model form of the equations are also given.
\end{abstract}

4.20.Me, 11.10.Lm, 11.30.Ly

Typeset using REVTEX 


\section{INTRODUCTION}

Integrable systems in two dimensions have been much studied in recent years and there are a number of books that discuss the developments [1 1 . Among the main results are the derivations of various integrable non-linear differential equations from zero curvature conditions. For example, it is known that the $\mathrm{KdV}$ hierarchy of equations may be derived from an $\mathrm{SL}(2, \mathrm{R})$ zero curvature condition in $1+1$ dimensions using a particular parametrization of the gauge field. It is also known that various other integrable equations may be similarly derived. This is because the zero curvature condition is the linearized Lax form of a two dimensional non-linear field equation [2], and it is this form which is essential for obtaining conserved quantities and proving that they are in involution.

More recent results show that many integrable equations are also derivable from selfduality conditions in four dimensions. For example, using again various parametrizations of an $\mathrm{SL}(2, \mathrm{R})$ gauge field, and this time imposing self-duality on the curvatures followed by a dimensional reduction to two dimensions, it has been shown that one can obtain the $\mathrm{KdV}$, Sine-Gordon, and non-linear Schrodinger equations [4] [6]. Furthermore, it has been shown recently that not just the $\mathrm{KdV}$ equation but the entire $\mathrm{KdV}$ hierarchy of equations may be obtained from an $\mathrm{SL}(2, \mathrm{R})$ self-duality condition in $2+2$ dimensions [7].

The two dimensional chiral model based on finite dimensional Lie groups is another example of a two dimensional integrable system [1]. One of its field equations is again a zero curvature condition for the relevant group. It is this model, but with an infinite dimensional Lie group, that will be of relevance for the self-dual Einstein equations (SDE) as discussed in this paper. It will turn out, via this analogy that the SDE may also be written as a zero curvature condition and one more equation.

Integrability of the two dimensional models mentioned above is intimately connected with the existense of 'hidden' symmetries which are manifested through the existence of unexpected conserved quantities, as was slowly unravelled first for the KdV equation. Unlike these models, however, the full Einstein equations contain very few hidden symmetries, apart 
from the built in diffeomorphisms. It has been shown that the only other symmetries are constant rescalings of the metric [15]. This suggests that the vacuum Einstein equations may not be integrable. A further consequence of this for recent approaches to canonical quantum gravity is the non-existence of fully gauge invariant observables for compact spatial topologies, at least for pure gravity, since if such observables existed, they would also be the conserved charges associated with hidden symmetries.

However, this state of affairs may not be the case for the much studied self-dual Einstein equation [8.9]. There are indications that this system is entirely integrable [10] although an infinite number of commuting conserved quantities have so far not been constructed. There are a number of interesting results associated with these equations. Using a form of the SDE [11] suggested by the Ashtekar Hamiltonian variables for general relativity [12], a connection with the self-dual Yang-Mills equation has been demonstrated [13]: the SDE may be obtained from a $0+1$ dimensional reduction of the self-dual Yang-Mills equation by an appropriate choice of gauge group. Another result is that the field equation for the continuum limit of the Toda model is the same as the SDE for a special ansatz for the metric [14]. A further connection with two dimensional theories has been the derivation of the Plebanski equation [9] for self-dual metrics from a large $\mathrm{N}$ limit of the $\mathrm{SU}(\mathrm{N})$ chiral model [16].

It is also relatively easy to find solutions of the SDE, particularly using their $3+1$ form suggested by the Ashtekar variables. Using a one-Killing field reduction of these equations (to a $2+1$ field theory), an infinite class of solutions have been found that are parametrized by elements of the $w_{\infty}$ algebra (which is the algebra of area preserving diffeomorphisms in two dimensions) [17].

These insights, together with the results on two dimensional models described above raise a number of questions regarding the integrability and quantization of the SDE: What is the Lax pair associated with this equation? Since the Lax pair follows from the existence of two distinct Hamiltonian formulations, what are the two symplectic forms? What are the explicit forms of the conserved quantities? Are they in involution? 
This paper addresses one aspect of these questions, and is concerned with elaborating on the connection between the SDE and two dimensional theories, with emphasis on conservation laws for the former. This will have implications for the existence of fully gauge invariant observables for the self-dual sector of the Einstein equations.

In the next section, we review the construction of non-local conservation laws from the field equations of the chiral model. Then in section three, we show how the SDE written in the $3+1$ form of Ashtekar, Jacobson and Smolin may be interpreted as equations for a two dimensional theory. It is shown how this interpretation allows, using the ideas of the second section, an explicit construction of an infinite number of conserved currents. In section four the SDE is written in a form that allows identification with the chiral model when the gauge group is the group of area preserving diffeomorphisms of a two dimensional surface. The method of section two is again applied to give the conserved currents explicitly. The last section contains a discussion and conclusions.

\section{CHIRAL MODEL AND CONSERVATION LAWS}

The chiral field $g(x, t)$ is a mapping from a $2 \mathrm{~d}$ spacetime into a group $g$. The dynamics follows from the Lagrangian density

$$
L=\frac{1}{2} \operatorname{Tr}\left(\partial_{\mu} g^{-1} \partial_{\nu} g\right) \eta^{\mu \nu}
$$

where $\eta^{\mu \nu}$ is the flat Minkowski or Euclidean metric. The equations of motion are

$$
\partial_{\mu}\left(g^{-1} \partial_{\mu} g\right)=0
$$

If we define the Lie algebra valued 1 -form $A_{\mu}:=g^{-1} \partial_{\mu} g$, then this equation of motion becomes

$$
\partial_{\mu} A_{\mu}=0
$$

Since $A_{\mu}$ by definition has a pure gauge form, it follows that 


$$
F_{\mu \nu}=\partial_{\mu} A_{\nu}-\partial_{\nu} A_{\mu}+\left[A_{\mu}, A_{\nu}\right]=0 .
$$

Associated with the gauge field $A_{\mu}$ there is also the covariant derivative

$$
D_{\mu}=\partial_{\mu}+\left[A_{\mu},\right]
$$

Thus the chiral model describes flat connections $A_{\mu}$ satisfying $\partial_{\mu} A_{\mu}=0$. Equations (2.3-2.4) are the first order forms of the field equation (2.2).

There exists a simple procedure that demonstrates the existence of an infinite number of non-local conserved currents starting from equations (2.3-2.4) [18].

Since the field equation $\partial_{\mu} A_{\mu}=0$ looks like a conservation law, define the first conserved current to be

$$
j_{\mu}^{(1)}(x):=A_{\mu}(x)
$$

This implies that there exists a function $f^{(1)}(x)$ such that

$$
j_{\mu}^{(1)}=\epsilon_{\mu}{ }^{\nu} \partial_{\nu} f^{(1)}
$$

where $\epsilon^{\mu \nu}$ is the Levi-Civita tensor. We now define the second current

$$
j_{\mu}^{(2)}:=D_{\mu} f^{(1)}
$$

It is easy to verify that it is conserved:

$$
\begin{aligned}
\partial_{\mu} j_{\mu}^{(2)} & =D_{\mu} \partial_{\mu} f^{(1)} \\
& =-\epsilon_{\mu}^{\alpha} D_{\alpha} j_{\mu}^{(1)}=-\epsilon_{\mu}^{\alpha} D_{\alpha} D_{\mu} f^{(0)} \\
& =-F_{01} f^{(0)}=0,
\end{aligned}
$$

where the first equality follows because $\partial_{\mu} A_{\mu}=0$, and $f^{(0)}=1$ because $j_{\mu}^{(1)} \equiv A_{\mu}$. Define now the $n$th current by

$$
j_{\mu}^{(n)}=D_{\mu} f^{(n-1)}
$$

Assuming it is conserved, implies there exists a function $f^{(n)}$ such that 


$$
j_{\mu}^{(n)}=\epsilon_{\mu}^{\nu} \partial_{\nu} f^{(n)}
$$

It follows that $j_{\mu}^{(n+1)}$ is conserved:

$$
\begin{aligned}
\partial_{\mu} j_{\mu}^{(n+1)} & =D_{\mu} \partial_{\mu} f^{(n)} \\
& =-\epsilon^{\alpha \mu} D_{\alpha} j_{\mu}^{(n)}=-\epsilon^{\alpha \mu} D_{\alpha} D_{\mu} f^{(n-1)} \\
& =-F_{01} f^{(n-1)}=0 .
\end{aligned}
$$

Thus the infinite set of currents $j_{\mu}^{(n)}(2.10)$ are conserved. Since the currents are built up recursively, the associated conserved charges $q^{(n)}$ are non-local. For example

$$
q^{(2)}(t)=\int_{-\infty}^{+\infty} d x j_{0}^{(2)}(x, t)=\int_{-\infty}^{+\infty} d x\left(\partial_{0}+A_{0}(x, t)\right) f^{(1)}(x, t)
$$

where

$$
f^{(1)}(x, t)=\int_{-\infty}^{x} d x^{\prime} A_{0}\left(t, x^{\prime}\right)
$$

follows from integrating (2.7). The integration constant only contributes a term proportional to the first conserved charge $q^{(1)}$.

In the following section it is shown how this procedure may be applied to the self-dual Einstein equation.

\section{SELF-DUAL EINSTEIN EQUATION}

The SDE may be written in a first order form using the Ashtekar Hamiltonian variables for general relativity [11].

Self-duality is the essential ingredient for this canonical formulation and it is natural to ask how the SDE looks in it. The phase space coordinate is the spatial projection of the (anti)self-dual part of the spin connection and its conjugate momentum is a densitized dreibein. The same is true for Euclidean or $(2,2)$ signatures, or complex general relativity, which are the cases of interest for self-dual Riemann curvatures. 
In these Hamiltonian variables, we would like to know what is the phase space condition corresponding to the vanishing of the (anti)self-dual part of the four dimensional Riemann curvature. The answer is that the spatial projection of the latter must be zero. The vanishing of this spatial projection, when substituted into Ashtekar's $3+1$ evolution equations leads to the new form of the SDE. It is straightforward to verify that this condition remains zero under the Hamiltonian evolution. The resulting equations on four-manifolds $M=\Sigma^{3} \times R$ may be written in terms of three spatial vector fields $V_{i}^{a}$ on $\Sigma^{3}$ :

$$
\begin{aligned}
\operatorname{Div}_{i}^{a} & =0 \\
\frac{\partial V_{i}^{a}}{\partial t} & =\frac{1}{2} \epsilon_{i j k}\left[V_{j}, V_{k}\right]^{a},
\end{aligned}
$$

where the divergence is defined with respect to a constant auxiliary density and the right hand side of (3.2) is the Lie bracket. The self-dual four metrics are constructed from solutions of these equations using

$$
g^{a b}=(\operatorname{det} V)^{-1}\left[V_{i}^{a} V_{j}^{b} \delta^{i j}+V_{0}^{a} V_{0}^{b}\right]
$$

Here $i, j, k \ldots=1,2,3$ label the vector field, $a, b, \ldots$ are abstract vector indices, $V_{0}^{a}$ is the vector field that is used to perform the $3+1$ decomposition, and $\partial V_{i}^{a} / \partial t \equiv V_{0}^{b} \partial_{b} V_{i}^{a}$. The time derivative in $(3.2)$ can be written in the more general form $\left[V_{0}, V_{i}\right]^{a}$. (For details of the derivation of these equations the reader is referred to [1] where they were originally derived, or the review in [17]).

Our starting point will be the SDE in the form (3.1-3.2). We first rewrite equation (3.2) in a form similar to that suggested by Yang [19 for the self-dual Yang-Mills equation

$$
F_{a b}=\frac{1}{2} \epsilon_{a b}^{c d} F_{c d}
$$

on a complex manifold. Replacing the (local) complex flat coordinates $x_{0}, \ldots, x_{3}$ by the linear combinations $t=x_{0}+i x_{1}, u=x_{0}-i x_{1}, x=x_{2}-i x_{3}$ and $v=x_{2}+i x_{3}$, equation (3.4) becomes

$$
\begin{aligned}
& F_{t x}=F_{u v}=0 \\
& F_{t u}+F_{x v}=0 .
\end{aligned}
$$


For the SDE, defining in a similar way

$$
\begin{array}{ll}
\mathcal{T}=V_{0}+i V_{1} & \mathcal{U}=V_{0}-i V_{1} \\
\mathcal{X}=V_{2}-i V_{3} & \mathcal{V}=V_{2}+i V_{3},
\end{array}
$$

the evolution equations (3.2) become

$$
\begin{aligned}
& {[\mathcal{T}, \mathcal{X}]=[\mathcal{U}, \mathcal{V}]=0} \\
& {[\mathcal{T}, \mathcal{U}]+[\mathcal{X}, \mathcal{V}]=0,}
\end{aligned}
$$

where the vector indices have been suppressed. This shows a rather direct analogy between the self-dual Yang-Mills and Einstein equations, namely, the Yang-Mills curvatures are replaced by the Lie brackets of the vector fields. A similar analogy has been noted in a related way in ref. [13.

We will now show that equations (3.8-3.9) give rise to an infinite number of conservation laws in a manner similar to that for the chiral model described in the last section. We fix (locally) a coordinate system $(t, x, p, q)$ and using the gauge freedom, fix in these coordinates

$$
\mathcal{T}^{a}=\left(\frac{\partial}{\partial t}\right)^{a} \quad \mathcal{X}^{a}=\left(\frac{\partial}{\partial x}\right)^{a}
$$

with $\mathcal{U}, \mathcal{V}$ arbitrary except that they be divergence free with respect to the volume form defined by the local coordinates: $\omega=d t \wedge d x \wedge d p \wedge d q$. Equations (3.8-3.9) then become

$$
\begin{gathered}
\frac{\partial \mathcal{U}}{\partial t}+\frac{\partial \mathcal{V}}{\partial x}=0 \\
{[\mathcal{U}, \mathcal{V}]=0}
\end{gathered}
$$

These are to be compared with equations (2.3-2.4) of the chiral model. The former has the form of a continuity equation on the two dimensional $(x, t)$ plane where there is a flat 2-d background metric $\delta_{\mu \nu}, \mu, \nu, \ldots=x, t$. Define a two dimensional vector field valued 1-form whose components are the vector fields $\mathcal{U}, \mathcal{V}:$ 


$$
A_{\mu}=(\mathcal{U} d t+\mathcal{V} d x)_{\mu}
$$

Equivalently, $A_{\mu}$ is a dyad whose components are vector fields. In this notation, we can rewrite equation (3.9) as $\delta^{\mu \nu} \partial_{\mu} A_{\nu}=0$ and so the first conserved current, by analogy with equation (2.6), is $J_{\mu}^{(1)}:=A_{\mu}$. Thus there exists a vector field $\eta^{(1)}$ such that

$$
J_{\mu}^{(1)}=\epsilon_{\mu}^{\nu} \partial_{\nu} \eta^{(1)}
$$

To avoid notational confusion, we emphasize that for the analogy with the chiral model equations, we are working in a fixed (local) coordinate system $t, x, p, q$, part of which $(t, x)$ is being interpreted as a two dimensional 'background' spacetime, and that the vector fields $\mathcal{U}, \mathcal{V}$ have components in this background, as well as in the 'internal' $(p, q)$ space. In addition, the vector fields $\mathcal{U}, \mathcal{V}$ are themselves the $(x, t)$ components of the vector field valued gauge field $A_{\mu}$ as defined in equation (3.13). Thus for example, (3.14) is an equation relating the components of the vector fields $J_{\mu}^{(1)}$ and $\eta^{(1)}$ in the given coordinate system.

Again following the analogy with the chiral model, we now define a second current and show that it is conserved. Since the analogy with the chiral model isn't exact, this definition is different from (2.8). Let

$$
J_{\mu}^{(2)}:=\left[A_{\mu}, \eta^{(1)}\right]
$$

We have

$$
\begin{aligned}
\delta^{\mu \nu} \partial_{\mu} J_{\nu}^{(2)} & =\delta^{\mu \nu} \partial_{\mu}\left[A_{\nu}, \eta^{(1)}\right]=\delta^{\mu \nu}\left[A_{\nu}, \partial_{\mu} \eta^{(1)}\right] \\
& =-\delta^{\mu \nu}\left[A_{\nu}, J_{\alpha}^{(1)}\right] \epsilon_{\mu}^{\alpha}=-2[\mathcal{U}, \mathcal{V}]=0
\end{aligned}
$$

by equation (3.12). Assuming now a conserved current

$$
J_{\mu}^{(n)}:=\left[A_{\mu}, \eta^{(n-1)}\right]=\epsilon_{\mu}{ }^{\nu} \partial_{\nu} \eta^{(n)}
$$

it is easy to show that

$$
J_{\mu}^{(n+1)}:=\left[A_{\mu}, \eta^{(n)}\right]
$$


is conserved:

$$
\begin{aligned}
\delta^{\mu \nu} \partial_{\mu} J_{\nu}^{(n+1)} & =\delta^{\mu \nu}\left[A_{\nu}, \partial_{\mu} \eta^{(n)}\right] \\
& =-\epsilon^{\mu \nu}\left[A_{\nu}, J_{\mu}^{(n)}\right]=-\epsilon^{\mu \nu}\left[A_{\nu},\left[A_{\mu}, \eta^{(n-1)}\right]\right] \\
& =-\left[\mathcal{U},\left[\mathcal{V}, \eta^{(n-1)}\right]\right]+\left[\mathcal{V},\left[\mathcal{U}, \eta^{(n-1)}\right]\right] \\
& =-\left[[\mathcal{U}, \mathcal{V}], \eta^{(n-1)}\right]=0
\end{aligned}
$$

Thus we have shown that equations (3.11-3.12) imply the conservation of the infinite

number of vector field valued currents $J_{\mu}^{(n)}$ defined in equation (3.17). All these currents are clearly independent, as for the chiral model case, by the recursive construction of equation (2.10) or (3.18). (We remind the reader that the two components $\mu=0,1$ of $A_{\mu}$ or $J_{\mu}^{(n)}$ corresponding to the $t, x$ coordinates, are themselves four dimensional vector fields, and all the above equations hold for each component of the vector fields in the coordinate system.)

The corresponding conserved charges are four dimensional vector fields. The components of the first two charges are given in terms of the integrals of the components of the vector field $\mathcal{U}$ using

$$
\begin{aligned}
Q^{(1)}(t) & =\int d x d p d q J_{0}^{(1)}=\int d x d p d q \mathcal{U} \\
Q^{(2)}(t) & =\int d x d p d q J_{0}^{(2)}=\int d x d p d q\left[\mathcal{U}, \int^{x} d x^{\prime} d p d q \mathcal{U}\left(t, x^{\prime}, p, q\right)\right]
\end{aligned}
$$

This shows the non-local nature of the charges. These are however only formal expressions, since for their evaluation for particular metrics, the integration limits will depend on the 'spatial' topologies.

\section{SELF-DUALITY EQUATIONS AS A CHIRAL MODEL}

In the last section we introduced a coordinate system and showed that the SDE implies an infinte set of conserved currents by using an analogy with the two dimensional chiral model. The SDE (3.11-3.12) in this coordinate system do not however take exactly the chiral model form. 
In this section we use a specific but general form for the vector fields $\mathcal{U}, \mathcal{V}$ and show that the SDE may be written exactly as the chiral model equations (2.3-2.4), with the gauge group being the group of area preserving diffeomorphisms of a two dimensional surface.

Before doing this we first give, for later comparison, the connection between the equations (3.11-3.12) and the Plebanski equation [9,20]. The vector fields $\mathcal{X}, \mathcal{T}$ as chosen in the above coordinate system are already divergence free with respect to the volume form $\omega$. We now take the vector fields $\mathcal{U}, \mathcal{V}$ to have the following form, such that they are divergence free, and are given in terms of one function $\Omega(t, x, p, q)$ :

$$
\begin{aligned}
& \mathcal{U}=-\Omega_{x q} \frac{\partial}{\partial p}+\Omega_{x p} \frac{\partial}{\partial q} \\
& \mathcal{V}=\Omega_{t q} \frac{\partial}{\partial p}-\Omega_{t p} \frac{\partial}{\partial q}
\end{aligned}
$$

where the subscripts denote partial differentiation. This identically satisfies equation (3.11), and equation (3.12) gives

$$
\Omega_{x p} \Omega_{t q}-\Omega_{x q} \Omega_{t p}=h(x, t),
$$

where $h(x, t)$ is an arbitrary function. With the change of variables $q \rightarrow q h(x, t)$, this becomes Plebanki's first equation

$$
\Omega_{x p} \Omega_{t q}-\Omega_{x q} \Omega_{t p}=1 .
$$

Furthermore, equation (3.3) for the metric leads to the line element

$$
d s^{2}=\Omega_{t p} d t d p+\Omega_{t q} d t d q+\Omega_{x p} d x d p+\Omega_{x q} d x d q
$$

We now show how the SDE may be written as a chiral model field equation. Again working in the above coordinate system, and with the same choice (3.10) for $\mathcal{T}, \mathcal{X}$, we take the following divergence free form $\mathbb{U}$ for $\mathcal{U}, \mathcal{V}$ in terms of the two functions $A_{0}(t, x, p, q)$ and $A_{1}(t, x, p, q)$ :

\footnotetext{
${ }^{1}$ This form is similar to, but more general than that used by the author previously in ref. [17], where one-Killing field reductions of the self-duality equations are discussed.
} 


$$
\begin{aligned}
\mathcal{U}^{a} & =\left(\frac{\partial}{\partial t}\right)^{a}+\alpha^{b a} \partial_{b} A_{0} \\
\mathcal{V}^{a} & =\left(\frac{\partial}{\partial x}\right)^{a}+\alpha^{b a} \partial_{b} A_{1},
\end{aligned}
$$

where $\alpha^{a b}=(\partial / \partial p)^{[a} \otimes(\partial / \partial q)^{b]}$ is the antisymmetric tensor that is the inverse of the two form $(d p \wedge d q)_{a b}$ in the $p, q$ plane. Substituting (4.6-4.7) into (3.11-3.12) gives

$$
\begin{gathered}
\alpha^{a b} \partial_{b}\left[\partial_{0} A_{1}-\partial_{1} A_{0}+\left\{A_{0}, A_{1}\right\}\right]=0 \\
\alpha^{a b} \partial_{b}\left[\partial_{0} A_{0}+\partial_{1} A_{1}\right]=0
\end{gathered}
$$

where the bracket on the left hand side of equation (4.8) is the Poisson bracket with respect to $\alpha^{a b}$ :

$$
\left\{A_{0}, A_{1}\right\}:=\alpha^{a b} \partial_{a} A_{0} \partial_{b} A_{1}=\partial_{p} A_{0} \partial_{q} A_{1}-\partial_{q} A_{0} \partial_{p} A_{1}
$$

and $\partial_{0}, \partial_{1}$ denote partial derivatives with respect to $t, x$ etc. Equations (4.8-4.9) imply that the terms in their square brackets are equal to two arbitrary functions of $t, x$, which we write as

$$
\begin{aligned}
\partial_{0} A_{1}-\partial_{1} A_{0}+\left\{A_{0}, A_{1}\right\} & =\partial_{0} F(t, x)+\partial_{1} G(t, x) \\
\partial_{0} A_{0}+\partial_{1} A_{1} & =\partial_{1} F(t, x)-\partial_{0} G(t, x),
\end{aligned}
$$

(where $F, G$ are arbitrary.) With the redefinitions

$$
a_{0}(t, x, p, q):=A_{0}+G \quad a_{1}(t, x, p, q):=A_{1}-F
$$

(4.11-4.12) become

$$
\begin{aligned}
\partial_{0} a_{1}-\partial_{1} a_{0}+\left\{a_{0}, a_{1}\right\} & =0 \\
\partial_{0} a_{0}+\partial_{1} a_{1} & =0 .
\end{aligned}
$$

These are precisely the chiral model equations (2.3-2.4) on the $x, t$ 'spacetime', with $p, q$ treated as coordinates on an internal space. The commutator has been replaced by the Poisson bracket (4.10). The gauge group is therefore the group of diffeomorphisms that 
preserve $\alpha^{a b}$ on the internal space. (Note that the redefinitions (4.13) do not alter the vector fields $\mathcal{U}, \mathcal{V}$ in equations $(4.6-4.7))$

Unlike in the last section, the procedure of section 2 for constructing conservation laws applies more directly to this case. The only difference is that the Lie group is now infinite dimensional. Denoting the pair $a_{0}, a_{1}$ by $a_{\mu}$, we have the covariant derivative

$$
D_{\mu}=\partial_{\mu}+\left\{a_{\mu},\right\}
$$

The first current is as before $J_{\mu}^{(1)}:=a_{\mu}$, which implies that there exists a function $g(t, x, p, q)$ such that $J_{\mu}^{(1)}=\epsilon_{\mu}{ }^{\nu} \partial_{\nu} g^{(1)}$. The $n$th conserved current is defined by

$$
J_{\mu}^{(n)}:=D_{\mu} g^{(n-1)}
$$

where like equation $(2.7), g^{(n-1)}$ is defined by $J_{\mu}^{(n-1)}=\epsilon_{\mu}{ }^{\nu} \partial_{\nu} g^{(n-1)}$. The inductive proof in section 2 of the conservation of these currents goes through unaltered.

The conserved charges are now integrals of the functions $a_{0}$. The first two are

$$
\begin{aligned}
& Q^{(1)}(t)=\int d x d p d q a_{0} \\
& Q^{(2)}(t)=\int d x d p d q\left[\partial_{0} g^{(1)}+\left\{a_{0}, g^{(1)}\right\}\right]
\end{aligned}
$$

where $g^{(1)}=\int^{x} d x^{\prime} d p d q a_{0}\left(t, x^{\prime}, p, q\right)$.

As contrasted with the last section where no fixed form of the vector fields $\mathcal{U}, \mathcal{V}$ is used, here we have the form (4.6-4.7) which leads to a specific internal gauge group. This group can fixed by choosing the 'internal' space to be some specific two-dimensional manifold $\Sigma$ and then one can try to solve the two dimensional equations (4.14-4.15). Since $a_{\mu}$ is valued in the Lie algebra of the area preserving diffeomorphisms, for compact internal spaces, such as $T^{2}$, the integrals over $p, q$ can be done explicitly by working in a specific basis for the Lie algebra. Such bases have been studied for a number of two surfaces [21]. We may further choose the space coordinatized by $x$ to be $S^{1}$ and seek solutions periodic in $x$. This will then allow an explicit evaluation of all the conserved charges for compact spatial three manifolds of topology $S^{1} \times \Sigma$. 
There is a further question regarding the relation between the full SDE (3.11-3.12) and the chiral model equations (4.14-4.15) derived from them. How general is the form (4.6-4.7) for the vector fields? We can see that the two first order equations for $a_{0}, a_{1}$ are equivalent to a single second order equation for a function $\Lambda(t, x, p, q)$ obtained by writing

$$
a_{0}=\partial_{1} \Lambda \quad a_{1}=-\partial_{0} \Lambda
$$

This solves equation (4.15) while equation (4.14) becomes

$$
\Lambda_{t t}+\Lambda_{x x}+\Lambda_{x p} \Lambda_{t q}-\Lambda_{x q} \Lambda_{t p}=0
$$

(where the subscripts denote partial derivatives.) This is one equation for a function of all the spacetime coordinates and therefore doesn't represent any reduction in the local degrees of freedom for self-dual metrics. Using equation (3.3) the line element is

$$
\begin{aligned}
d s^{2}= & -d t\left(\Lambda_{t p} d p+\Lambda_{t q} d q\right)-d x\left(\Lambda_{x p} d p+\Lambda_{x q} d q\right) \\
& +\frac{1}{\left\{\Lambda_{t}, \Lambda_{x}\right\}}\left(\left(\Lambda_{x p} d p+\Lambda_{x q} d q\right)^{2}+\left(\Lambda_{t p} d p+\Lambda_{t q} d q\right)^{2}\right)
\end{aligned}
$$

Equation (4.21) is essentially another way of writing the SDE, which is different from Plebanski's two equations.'2 That it has the same content is seen most simply by comparing the forms of the vector fields $\mathcal{U}, \mathcal{V}$ (4.1-4.2), which give the first Plebanski equation, with the following final form of the vector fields (obtained by substituting (4.20) into (4.6-4.7)), which give the alternative equation (4.21):

$$
\begin{aligned}
& \mathcal{U}=\frac{\partial}{\partial t}-\Omega_{x q} \frac{\partial}{\partial p}+\Omega_{x p} \frac{\partial}{\partial q} \\
& \mathcal{V}=\frac{\partial}{\partial x}+\Omega_{t q} \frac{\partial}{\partial p}-\Omega_{t p} \frac{\partial}{\partial q}
\end{aligned}
$$

\footnotetext{
${ }^{2}$ Another alternative to the Plebanski equation has also been discussed recently [24]. This approach also starts from the Ashtekar-Jacobson-Smolin equations but the remaining development is different from that given here, as is the final equation.
} 
The forms of the vector fields (4.1-4.2) and (4.23-4.24) clearly have the same functional content.

The usefulness of equation (4.21) is its direct connection with the chiral model, which leads easily to the conservation laws and the Hamiltonian formulation. The latter is given in ref. [1] for finite dimensional groups, and this immediately generalizes to the infinite dimensional case.

\section{CONCLUSIONS AND DISCUSSION}

There are two main results presented in this paper. The first provides a simple way to view the SDE as the field equation of a two dimensional theory, the chiral model with gauge group the group of area preserving diffeomorphisms of a two dimensional surface. The second result is an explicit construction of an infinite number of conserved currents for the SDE, which was presented here in two different ways in sections 3 and 4 . f

The first result has been noted before in a different context in [16] where the inverse scattering form of the chiral model equations were related to Plebanski's first equation. Here, on the other hand, we have seen that the explicit chiral model equations (4.14-4.15) may be derived from the Ashtekar-Jacobson-Smolin form of the SDE, and that its second order form leads to equation (4.21), which is different from the two Plebanski heavenly equations.

The second result shows that, unlike the full Einstein equations, the self-dual sector is rather rich in conservation laws. This has implications for the quantum theory for this sector, since in the canonical approach to quantum gravity, one of the goals is to identify

\footnotetext{
${ }^{3} \mathrm{~A}$ recent paper also discusses non-local conservation laws for the SDE using a completely different twistor approach [23]. The method makes use of the connection between the null surfaces corresponding to self-dual spaces and the Plebanski equation. It would be of interest to compare this to the approach given above.
} 
fully gauge invariant phase space functionals ('observables'), which are to be represented as Hermitian operators on the Hilbert space. This has been a stumbling block for full Einstein gravity where no such observables are known. The self-dual sector may be viewed as perhaps the largest possible midi-superspace (albeit with the wrong signature metric), but the results given here show that its phase space has an infinite number of observables.

A further implication of these results is that, unlike the Plebanski heavenly equations, equation (4.21) has the advantage that it has an obvious Hamiltonian formulation - that of the two dimensional chiral model. In particular it should be possible to rewrite the conserved charges in terms of Hamiltonian variables and compute explicitly their Poisson brackets. An important question for integrability is whether these charges are in involution. But whether or not they are in involution, we still have an infinite number of observables at hand for canonical quantization.

All known integrable two dimensional models have two Hamiltonian structures. The two symplectic forms provide an elegant way to generate all the conserved charges. An interesting question in this context is whether the SDE has two symplectic forms, and whether the conserved charges given here may be derived using these forms in the standard way [2]. The answer to this may be provided by asking what the two symplectic forms are for the chiral model.

An important result from the study of two dimensional models is that the transfer matrix, which is the path ordered exponential of the connection in the zero curvature form of the model, provides a way of constructing the conserved quantities. The connection in the zero curvature condition normally depends on the spectral parameter, and therefore the trace of the transfer matrix depends on it as well. This trace serves as the generating function for the conservation laws. Namely, the coefficients of powers of the spectral parameter in the expansion of the trace are the conserved charges [1,2]. (This procedure has recently been applied to the two Killing field reductions of the Einstein equations [22].)

On the other hand, here we have derived the conserved charges, which have a resemblance to terms in the expansion of such a trace, (the expansion of the Wilson loop for example.) 
However, it is not known how to construct the holonomy associated with an infinite dimensional group, let alone take its trace. In this regard it may be possible to invert the transfer matrix method of obtaining conserved charges, and use the latter obtained here to get an expansion for the holonomy associated with an infinite dimensional Lie group. Work on this and on the canonical quantization of the SDE using the above ideas is in progress [25].

\section{ACKNOWLEDGMENTS}

This work was supported by NSERC of Canada. 


\section{REFERENCES}

[1] L. D. Faddeev and L. A. Takhtajan, Hamiltonian methods in the theory of solitons, Springer (Berlin 1987).

[2] A. Das, Integrable models, World Scientific (Singapore 1989).

[3] A. C. Newell, Solitons in mathematical physics, SIAM (1985).

[4] R. S. Ward, Nucl. Phys. B236, 381 (1984).

[5] L. J. Mason and G. A. J. Sparling, Phys. Lett. A137, 29 (1989).

[6] I. Bakas and D. A. Depireux, Int. J. Mod. Phys. A7, 1767 (1992).

[7] A. Das and C. A. P. Galvao, Rochester preprint UR-1274 (1992).

[8] M. Ludvigsen, E.T. Newman and K. P. Tod, Phys. Rep. 71, 51 (1981).

[9] J. Plebanski, J. Math. Phys. 16, 2395 (1975).

[10] R. Penrose, Gen. Rel and Grav., 7, 31 (1976).

[11] A. Ashtekar, T Jacobson, and L. Smolin, Comm. Math. Phys. 115, 631 (1988).

[12] A. Ashtekar, Phys. Rev. Lett. 57, 2244 (1986); Phys. Rev. D36, 1587 (1987).

[13] L. Mason and E. T. Newman, Comm. Math. Phys. 121, 659, (1989).

[14] I. Bakas and E. Kiritsis, in Topological methods in field theory, ed. W. Nahm, S. Randjbar-Daemi, E. Sezgin and E. Witten, (World Scientific, Singapore 1991).

[15] C. G. Torre and I. M. Anderson, Phys. Rev. Lett. 70, 3525 (1993).

[16] Q. H. Park, Phys. Lett. B236, 429 (1990); Phys. Lett. B238, 287 (1990).

[17] V. Husain, Class. and Quantum Grav. 10, 543 (1993).

[18] E. Brezin, C. Itzykson, J. Zinn-Justin, and J. B. Zuber, Phys. Lett. B82, 442 (1979). 
[19] C. N. Yang, Phys. Rev. Lett.38, 1377 (1977).

[20] S. Chakravarty, L. Mason, and E. T. Newman, J. Math. Phys. 32, 1458 (1991).

[21] C. N. Pope and L. J. Romans, Class. Quantum. Grav 7, 97 (1990).

[22] N. Manojlovic and B. Spence, 'Integrals of motion in the two Killing vector reduction of general relativity', Syracuse preprint SU-GP-93/7-8.

[23] T. A. B. Strachan, Class. Quantum Grav. 10, 1417 (1993).

[24] J. D. E. Grant, Preprint DAMPT-R92/47.

[25] V. Husain and N. Manojlovic, work in progress. 